\title{
On Perturbative Gravity and Gauge Theory*
}

\author{
Z. Bern, ${ }^{a \dagger}$ L. Dixon, ${ }^{b}$ D.C. Dunbar, ${ }^{c}$ A.K. Grant, ${ }^{\mathrm{d}}$ M. Perelstein, ${ }^{\mathrm{b}}$ and J.S. Rozowsky ${ }^{\mathrm{e}}$ \\ a Department of Physics, UCLA, Los Angeles, CA 90095 \\ bStanford Linear Accelerator Center, Stanford University, Stanford, CA 94309 \\ cDepartment of Physics, University of Wales Swansea, Swansea SA2 8PP, UK \\ d Department of Physics, Harvard University, Cambridge, MA 02138 \\ eInstitute for Fundamental Theory, Department of Physics, University of Florida, Gainesville, FL 32611
}

We review some applications of tree-level (classical) relations between gravity and gauge theory that follow from string theory. Together with $D$-dimensional unitarity, these relations can be used to perturbatively quantize gravity theories, i.e. they contain the necessary information for obtaining loop contributions. We also review recent applications of these ideas showing that $N=1 D=11$ supergravity diverges, and review arguments that $N=8 D=4$ supergravity is less divergent than previously thought, though it does appear to diverge at five loops. Finally, we describe field variables for the Einstein-Hilbert Lagrangian that help clarify the perturbative relationship between gravity and gauge theory.

\section{Introduction}

In this talk we describe recent work [1] 5 on perturbative relations between gravity and gauge theories. Although gauge and gravity theories are similar in that they both have a local symmetry, their dynamical behavior is quite different. For example, in four dimensions gauge theories are renormalizable and exhibit asymptotic freedom, neither of which property is shared by gravity. The structures of the Lagrangians are also rather different: the Yang-Mills Lagrangian contains only up to four-point interactions while the Einstein-Hilbert Lagrangian contains infinitely many interactions. Nevertheless, in the context of perturbation theory, it turns out that tree-level gravity amplitudes can, roughly speaking, be expressed as a sum of 'squares' of gauge theory amplitudes. These tree-level (classical) relations between gravity and gauge theory amplitudes follow from the Kawai, Lewellen and Tye (KLT) 6] relations between open and closed string tree ampli-

\footnotetext{
${ }^{*}$ This research was supported by the US Department of Energy under grants DE-FG03-91ER40662, DE-AC0376SF00515 and DE-FG02-97ER41029.

${ }^{\dagger}$ Presenter at Third Meeting on Constrained Dynamics and Quantum Gravity, Villasimius (Sardinia, Italy) September 13-17, 1999 and at the Workshop on LightCone QCD and Nonperturbative Hadron Physics, University of Adelaide (Australia) December 13-22, 1999.
}

tudes. When combined with the $D$-dimensional unitarity methods described in refs. [7,8], it provides a useful tool for investigating the ultraviolet behavior of gravity field theories. The unitarity methods have also been applied to QCD loop computations of phenomenological interest and to supersymmetric gauge theory computations [7,26].

Ultraviolet properties are one of the central issues for perturbative gravity. Although gravity is non-renormalizable by power counting, no divergence has, in fact, been established by a direct calculation for any supersymmetric theory of gravity in four dimensions. Explicit calculations have established that non-supersymmetric theories of gravity with matter generically diverge at one loop [9 11], and pure gravity diverges at two loops 12$]$. However, in any supergravity theory in $D=4$, supersymmetry Ward identities 13 forbid all possible one-loop [14] and two-loop 15] counterterms. Thus, at least a three-loop calculation is required to definitively address the question of divergences in four-dimensional supergravity. There is a candidate counterterm at three loops for all supergravities including the maximally extended version $(N=8)$ 16, 17. However, no explicit three loop (super) gravity calculations have appeared. It is in principle possible that the coefficient of a potential counterterm can vanish, es- 


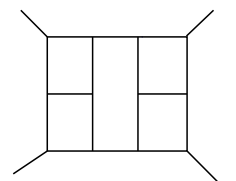

Figure 1. An example of a five-loop diagram.

pecially if the full symmetry of the theory is taken into account. In ref. [1] it was argued that this may indeed be the case for the potential threeloop counterterm of $N=8 D=4$ supergravity. For there to be cancellations of divergences beyond this, the theory would require a symmetry which we do not fully understand yet.

With traditional perturbative approaches 18 to performing explicit calculations, as the number of loops increases the number of algebraic terms proliferates rapidly beyond the point where computations are practical. Consider the five-loop diagram in fig. 11 (which, as described below, is of interest for ultraviolet divergences in $N=8$ $D=4$ supergravity). In de Donder gauge the pure graviton diagram contains twelve vertices, each of the order of a hundred terms, and sixteen graviton propagators, each with three terms. When expanded, this yields a total of roughly $10^{30}$ terms, even before performing any loop integrations. Needless to say, this is well beyond what can be reasonably implemented on any computer.

Our approach for dealing with this is to reformulate the quantization of gravity in the context of perturbation theory using the KLT relations together with $D$-dimensional unitarity.

The final topic of this talk will cover some recent progress in understanding the KLT relations

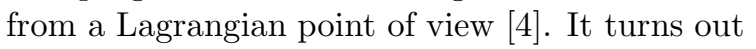
that with an appropriate choice of field variables one can separate the Lorentz indices appearing in the Lagrangian into 'left' and 'right' classes [19.4], mimicking the similar separation that occurs in string theory. Moreover, with further field redefinitions and a non-linear gauge choice it is possible to arrange the off-shell three graviton vertex so that it is expressible in terms of a sum of squares of Yang-Mills three gluon vertices.

\section{Method for Investigating Perturbative Gravity}

Our formulation of perturbative quantum gravity is based on two ingredients:

1. The Kawai, Lewellen and Tye relations be- tween closed and open string tree-level Smatrices [6].

2. The observation that the $D$-dimensional tree amplitudes contain all information necessary for building the complete perturbative $S$-matrix to all loop orders [7]:8].

\subsection{The KLT tree-level relations.}

In the field theory limit $\left(\alpha^{\prime} \rightarrow 0\right)$ the KLT relations for the four- and five-point amplitudes are 20

$$
\begin{aligned}
& M_{4}^{\text {tree }}(1,2,3,4)= \\
& \quad-i s_{12} A_{4}^{\text {tree }}(1,2,3,4) A_{4}^{\text {tree }}(1,2,4,3), \\
& M_{5}^{\text {tree }}(1,2,3,4,5)= \\
& \quad i s_{12} s_{34} A_{5}^{\text {tree }}(1,2,3,4,5) A_{5}^{\text {tree }}(2,1,4,3,5) \\
& +i s_{13} s_{24} A_{5}^{\text {tree }}(1,3,2,4,5) A_{5}^{\text {tree }}(3,1,4,2,5),
\end{aligned}
$$

where the $M_{n}$ 's are the amplitudes in a gravity theory stripped of couplings, the $A_{n}$ 's are the color-ordered sub-amplitudes in a gauge theory and $s_{i j} \equiv\left(k_{i}+k_{j}\right)^{2}$. We suppress all $\varepsilon_{j}$ polarizations and $k_{j}$ momenta, but keep the ' $j$ ' labels to distinguish the external legs. Full gauge theory amplitudes are given in terms of the partial amplitudes $A_{n}$, via

$$
\begin{aligned}
\mathcal{A}_{n}^{\text {tree }}(1,2, \ldots n)=g^{(n-2)} & \sum_{\sigma \in S_{n} / Z_{n}} \\
& \operatorname{Tr}\left(T^{a_{\sigma(1)}} \ldots T^{a_{\sigma(n)}}\right) A_{n}^{\text {tree }}(\sigma(1), \ldots, \sigma(n)),
\end{aligned}
$$

where $S_{n} / Z_{n}$ is the set of all permutations, but with cyclic rotations removed, and $g$ is the gauge theory coupling constant. The $T^{a_{i}}$ are fundamental representation matrices for the YangMills gauge group $S U\left(N_{c}\right)$, normalized so that $\operatorname{Tr}\left(T^{a} T^{b}\right)=\delta^{a b}$. For states coupling with the strength of gravity, the full amplitudes including the gravitational coupling constant are,

$\mathcal{M}_{n}^{\text {tree }}(1, \ldots n)=\left(\frac{\kappa}{2}\right)^{(n-2)} M_{n}^{\text {tree }}(1, \ldots n)$,

where $\kappa^{2}=32 \pi G_{N}$. The KLT equations generically hold for any closed string states, using their Fock space factorization into pairs of open string states.

Berends, Giele and Kuijf [20] exploited the KLT relations (11) and their $n$-point generalizations to obtain an infinite set of maximally helicity violating (MHV) graviton tree amplitudes, using the known MHV Yang-Mills amplitudes [21]. Cases of gauge theory coupled to gravity have 
very recently been discussed in ref. [5]. Interestingly, the color charges associated with any gauge fields appearing in gravity theories are represented through the KLT equations as flavor charges carried either by scalars or fermions. For example, by applying the KLT equations the three-gluon one-graviton amplitude may be expressed as

$$
\begin{aligned}
& \mathcal{M}_{4}^{\text {tree }}\left(1_{g}^{-}, 2_{g}^{-}, 3_{g}^{+}, 4_{h}^{+}\right)=-i g \frac{\kappa}{2} s_{12} \\
& \quad \times A_{4}^{\text {tree }}\left(1_{g}^{-}, 2_{g}^{-}, 3_{g}^{+}, 4_{g}^{+}\right) \times A_{4}^{\text {tree }}\left(1_{s}, 2_{s}, 4_{g}^{+}, 3_{s}\right) \\
& =g \frac{\kappa}{2} \frac{\langle 12\rangle^{4}}{\langle 12\rangle\langle 23\rangle\langle 34\rangle\langle 41\rangle} \times \sqrt{2} f^{a_{1} a_{2} a_{3}} \frac{[43]\langle 32\rangle}{\langle 24\rangle},
\end{aligned}
$$

where the \pm superscripts denote the helicities and the subscripts $h, g$ and $s$ denote whether a given leg is a graviton, gluon or scalar. On the righthand side of the equation, the group theory indices are flavor indices for the scalars. On the lefthand side they are reinterpreted as color indices for gluons. For simplicity, the amplitudes have been expressed in terms of $D=4$ spinor inner products (see e.g. ref. [22]), although the factorization of the amplitude into purely gauge theory amplitudes holds in any dimension. The spinor inner products are denoted by $\langle i j\rangle=\left\langle i^{-} \mid j^{+}\right\rangle$ and $[i j]=\left\langle i^{+} \mid j^{-}\right\rangle$, where $\left|i^{ \pm}\right\rangle$are massless Weyl spinors of momentum $k_{i}$, labeled with the sign of the helicity. They are antisymmetric, with norm $|\langle i j\rangle|=|[i j]|=\sqrt{s_{i j}}$.

\subsection{Cut Construction of Loop Amplitudes}

We now outline the use of the KLT relations for computing multi-loop gravity amplitudes, starting from gauge theory amplitudes. Although the KLT equations hold only at the classical treelevel, $D$-dimensional unitarity considerations can be used to extend them to the quantum level. The application of $D$-dimensional unitarity has been extensively discussed for the case of gauge theory amplitudes [7,8] so here we describe it only briefly.

The unitarity cuts of a loop amplitude can be expressed in terms of amplitudes containing fewer loops. For example, the two-particle cut of a oneloop four-point amplitude in the channel carrying momentum $k_{1}+k_{2}$, as shown in fig. 2, can be expressed as the cut of,

$$
\begin{aligned}
\sum_{\text {states }} \int \frac{d^{D} L_{1}}{(4 \pi)^{D}} \frac{i}{L_{1}^{2}} \mathcal{M}_{4}^{\text {tree }}\left(-L_{1}, 1,2, L_{3}\right) \\
\times\left.\frac{i}{L_{3}^{2}} \mathcal{M}_{4}^{\text {tree }}\left(-L_{3}, 3,4, L_{1}\right)\right|_{\text {cut }},
\end{aligned}
$$

where $L_{3}=L_{1}-k_{1}-k_{2}$, and the sum runs

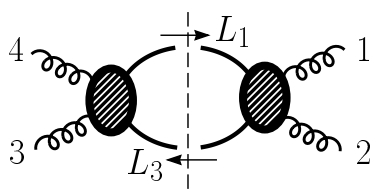

Figure 2. The two-particle cut at one loop in the channel carrying momentum $k_{1}+k_{2}$.

over all states crossing the cut. We label $D$ dimensional momenta with capital letters and four-dimensional ones with lower case. We apply the on-shell conditions $L_{1}^{2}=L_{3}^{2}=0$ to the amplitudes appearing in the cut even though the loop momentum is unrestricted; only functions with a cut in the given channel under consideration are reliably computed in this way.

Complete amplitudes are found by combining all cuts into a single function with the correct cuts in all channels. If one works with an arbitrary dimension $D$ in eq. (2), and takes care to keep the full analytic behavior as a function of $D$, then the results will be free of subtraction ambiguities that are commonly present in cutting methods [23,7, 8]. (The regularization scheme dependence remains, of course.)

An important advantage of the cutting approach is that the gauge-invariant amplitudes on either side of the cut may be simplified before attempting to evaluate the cut integral [8].

\section{Recycling Gauge Theory Into Gravity Loop amplitudes}

Consider the one-loop amplitude with four identical helicity gravitons and a scalar in the loop [2.3]. The cut in the $s_{12}$ channel is

$$
\begin{aligned}
\int \frac{d^{D} L_{1}}{(2 \pi)^{D}} \frac{i}{L_{1}^{2}} M_{4}^{\text {tree }} & \left(-L_{1}^{s}, 1^{+}, 2^{+}, L_{3}^{s}\right) \frac{i}{L_{3}^{2}} \\
& \times\left. M_{4}^{\text {tree }}\left(-L_{3}^{s}, 3^{+}, 4^{+}, L_{1}^{s}\right)\right|_{\text {cut }}
\end{aligned}
$$

where the superscript $s$ indicates that the cut lines are scalars. Using the KLT expressions (1) we may replace the gravity tree amplitudes appearing in the cuts with products of gauge theory amplitudes. The required gauge theory tree amplitudes, with two external scalar legs and two gluons, are relatively simple to obtain using Feyn- 
man diagrams and are,

$$
\begin{aligned}
& A_{4}^{\text {tree }}\left(-L_{1}^{s}, 1^{+}, 2^{+}, L_{3}^{s}\right)=i \frac{\mu^{2}[12]}{\langle 12\rangle\left[\left(\ell_{1}-k_{1}\right)^{2}-\mu^{2}\right]}, \\
& A_{4}^{\text {tree }}\left(-L_{1}^{s}, 1^{+}, L_{3}^{s}, 2^{+}\right)=-i \frac{\mu^{2}[12]}{\langle 12\rangle} \\
& \quad \times\left[\frac{1}{\left(\ell_{1}-k_{1}\right)^{2}-\mu^{2}}+\frac{1}{\left(\ell_{1}-k_{2}\right)^{2}-\mu^{2}}\right]
\end{aligned}
$$

where $L_{1}=\ell_{1}+\mu$. The gluon momenta are fourdimensional, but the scalar momenta are allowed to have a $(-2 \epsilon)$-dimensional component $\vec{\mu}$, with $\vec{\mu} \cdot \vec{\mu}=\mu^{2}>0$. The overall factor of $\mu^{2}$ appearing in these tree amplitudes means that they vanish in the four-dimensional limit, in accord with a supersymmetry Ward identity [13]. In the KLT relation (11), one of the propagators cancels, leaving

$$
\begin{aligned}
M_{4}^{\text {tree }}( & \left.-L_{1}^{s}, 1^{+}, 2^{+}, L_{3}^{s}\right)=-i\left(\frac{\mu^{2}[12]}{\langle 12\rangle}\right)^{2} \\
& \times\left[\frac{1}{\left(\ell_{1}-k_{1}\right)^{2}-\mu^{2}}+\frac{1}{\left(\ell_{1}-k_{2}\right)^{2}-\mu^{2}}\right] .
\end{aligned}
$$

By symmetry, the tree amplitudes appearing in any of the other cuts are the same up to relabelings.

Combining all three cuts into a single function that has the correct cuts in all channels yields

$$
\begin{gathered}
M_{4}^{1-\text { loop }}\left(1^{+}, 2^{+}, 3^{+}, 4^{+}\right)=2 \frac{[12]^{2}[34]^{2}}{\langle 12\rangle^{2}\langle 34\rangle^{2}} \\
\times\left(\mathcal{I}_{4}^{1-\text { loop }}\left[\mu^{8}\right](s, t)+\mathcal{I}_{4}^{1-\text { loop }}\left[\mu^{8}\right](s, u)\right. \\
\left.+\mathcal{I}_{4}^{1-\operatorname{loop}}\left[\mu^{8}\right](t, u)\right),
\end{gathered}
$$

where $s=s_{12}, t=s_{14}, u=s_{13}$ are the usual Mandelstam variables and

$$
\begin{aligned}
& \mathcal{I}_{4}^{1 \text {-loop }}[\mathcal{P}](s, t)=\int \frac{d^{D} L}{(2 \pi)^{D}} \\
& \quad \times \frac{\mathcal{P}}{L^{2}\left(L-k_{1}\right)^{2}\left(L-k_{1}-k_{2}\right)^{2}\left(L+k_{4}\right)^{2}}
\end{aligned}
$$

is the scalar box integral depicted in fig. 3 with the external legs arranged in the order 1234 . In eq. (3) the numerator $\mathcal{P}$ is $\mu^{8}$. The two other scalar integrals that appear correspond to the two other distinct orderings of the four external legs. The spinor factor $[12]^{2}[34]^{2} /\left(\langle 12\rangle^{2}\langle 34\rangle^{2}\right)$ in eq. (3) is actually completely symmetric, although not manifestly so. By rewriting this factor and extracting the leading $\mathcal{O}\left(\epsilon^{0}\right)$ contribution

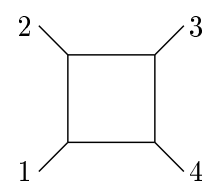

Figure 3. The one loop box integral.

from the integral, the final one-loop $D=4$ result after reinserting the gravitational coupling is

$$
\begin{aligned}
\mathcal{M}_{4}^{1 \text { loop }} & \left(1^{+}, 2^{+}, 3^{+}, 4^{+}\right)=-\frac{i}{(4 \pi)^{2}}\left(\frac{\kappa}{2}\right)^{4} \\
& \times\left(\frac{s t}{\langle 12\rangle\langle 23\rangle\langle 34\rangle\langle 41\rangle}\right)^{2} \frac{s^{2}+t^{2}+u^{2}}{120},
\end{aligned}
$$

in agreement with a previous calculation [24].

This can be extended to an arbitrary number of external legs. Using the cutting methods we have also calculated the five- and six-point amplitudes. By demanding that the amplitudes have the correct factorization properties as momenta become either soft or collinear [7,25] we have found an ansatz for the one-loop maximally helicity violating amplitudes for an arbitrary number of external legs [2, 3],

$$
\begin{aligned}
& \mathcal{M}_{n}^{1-\operatorname{loop}}\left(1^{+}, 2^{+}, \ldots, n^{+}\right)=-\frac{i}{(4 \pi)^{2} \cdot 960}\left(\frac{-\kappa}{2}\right)^{n} \\
& \quad \times \sum_{\substack{1 \leq a<b \leq n \\
M, N}} h(a, M, b) h(b, N, a) \operatorname{tr}^{3}[a M b N]
\end{aligned}
$$

where $a$ and $b$ are massless legs, and $M$ and $N$ are two sets forming a distinct nontrivial partition of the remaining $n-2$ legs. Also, $\operatorname{tr}[a M b N] \equiv$ $\operatorname{tr}\left[k_{a} K_{M} \not k_{b} K_{N}\right]$, where $K_{M}$ is the sum of momenta of the legs in the set $M$. The rational function $h$ is a bit more complicated and may be found in refs. 2, 3,

These amplitudes are not ultraviolet divergent and thus do not depend on a cutoff. By unitarity considerations any fundamental theory of gravity must therefore necessarily yield the one-loop amplitudes (5) and (6) in the low energy limit. (The tree amplitude can, however, have a contribution of the same dimension as that of the oneloop amplitude; this contribution would depend on the details of the underlying fundamental theory.) The ability to obtain exact expressions for gravity loop amplitudes demonstrates the utility of this approach for investigating quantum properties of gravity theories. As our next example, we turn to the divergence properties of maximally 
supersymmetric theories.

\section{Maximal Supergravity}

Maximal $N=8$ supergravity can be expected to be the least divergent of the four-dimensional supergravity theories due to its high degree of symmetry. Moreover, from a technical viewpoint maximally supersymmetric $N=8$ amplitudes are by far the easiest to deal with in our formalism because of spectacular supersymmetric cancellations. For these reasons it is logical to re-investigate the divergence properties of this theory first [1]. It should be possible to apply similar methods to theories with less supersymmetry.

\subsection{Cut Construction}

Again we obtain supergravity amplitudes by recycling gauge theory calculations. For the $N=8$ case, we factorize each of the 256 states of the multiplet into a tensor product of $N=4$ superYang-Mills states. The key equation for obtaining the two-particle cuts is,

$$
\begin{aligned}
& \sum_{\substack{N=8 \\
\text { states }}} M_{4}^{\text {tree }}\left(-L_{1}, 1,2, L_{3}\right) \times M_{4}^{\text {tree }}\left(-L_{3}, 3,4, L_{1}\right) \\
& =s^{2} \sum_{\substack{N=4 \\
\text { states }}} A_{4}^{\text {tree }}\left(-L_{1}, 1,2, L_{3}\right) \times A_{4}^{\text {tree }}\left(-L_{3}, 3,4, L_{1}\right) \\
& \quad \times \sum_{\substack{N=4 \\
\text { states }}} A_{4}^{\text {tree }}\left(L_{3}, 1,2,-L_{1}\right) \times A_{4}^{\text {tree }}\left(L_{1}, 3,4,-L_{3}\right),
\end{aligned}
$$

where the sum on the left-hand side runs over all states in the $N=8$ supergravity multiplet. On the right-hand side the two sums run over the states of the $N=4$ super-Yang-Mills multiplet: a gluon, four Weyl fermions and six real scalars. Given the corresponding $N=4$ Yang-Mills twoparticle sewing equation 26],

$$
\begin{aligned}
& \sum_{\substack{N=4 \\
\text { states }}} A_{4}^{\text {tree }}\left(-L_{1}, 1,2, L_{3}\right) \times A_{4}^{\text {tree }}\left(-L_{3}, 3,4, L_{1}\right) \\
& \quad=-i s t A_{4}^{\text {tree }}(1,2,3,4) \frac{1}{\left(L_{1}-k_{1}\right)^{2}} \frac{1}{\left(L_{3}-k_{3}\right)^{2}},
\end{aligned}
$$

it is a simple matter to evaluate eq. (7), yielding

$$
\begin{aligned}
& \sum_{\substack{N=8 \\
\text { states }}} M_{4}^{\text {tree }}\left(-L_{1}, 1,2, L_{3}\right) \times M_{4}^{\text {tree }}\left(-L_{3}, 3,4, L_{1}\right) \\
& =i s t u M_{4}^{\text {tree }}(1,2,3,4)\left[\frac{1}{\left(L_{1}-k_{1}\right)^{2}}+\frac{1}{\left(L_{1}-k_{2}\right)^{2}}\right] \\
& \times\left[\frac{1}{\left(L_{3}-k_{3}\right)^{2}}+\frac{1}{\left(L_{3}-k_{4}\right)^{2}}\right] .
\end{aligned}
$$

The sewing equations for the $t$ and $u$ channels are similar to that of the $s$ channel.

A remarkable feature of the cutting equation (8) is that the external-state dependence of the right-hand side is entirely contained in the tree amplitude $M_{4}^{\text {tree }}$. This fact allows us to iterate the two-particle cut algebra to all loop orders! Although this is not sufficient to determine the complete multi-loop four-point amplitudes, it does provide a wealth of information.

Applying eq. (8) at one loop to each of the three channels yields the one-loop four graviton amplitude of $N=8$ supergravity,

$$
\begin{gathered}
\mathcal{M}_{4}^{1 \text {-loop }}(1,2,3,4)=-i\left(\frac{\kappa}{2}\right)^{4} s t u M_{4}^{\text {tree }}(1,2,3,4) \\
\times\left(\mathcal{I}_{4}^{1 \text {-loop }}(s, t)+\mathcal{I}_{4}^{1 \text {-loop }}(s, u)+\mathcal{I}_{4}^{1-\operatorname{loop}}(t, u)\right),
\end{gathered}
$$

in agreement with previous results [27]. We have reinserted the gravitational coupling $\kappa$ in this expression. The scalar integrals are defined in eq. (4) with $\mathcal{P}=1$.

At two loops, the two-particle cuts are given by a simple iteration of the one-loop calculation. The three-particle cuts can be obtained by recycling the corresponding cuts for the case of $N=4$ super-Yang-Mills. It turns out that the three-particle cuts introduce no other functions than those already detected in the two-particle cuts. Combining all the cuts into a single function yields the $N=8$ supergravity two-loop amplitude [1],

$$
\begin{gathered}
\mathcal{M}_{4}^{2 \text {-loop }}(1,2,3,4)=\left(\frac{\kappa}{2}\right)^{6} \operatorname{stu}_{4}^{\text {tree }}(1,2,3,4) \\
\times\left(s^{2} \mathcal{I}_{4}^{2 \text {-loop }, \mathrm{P}}(s, t)+s^{2} \mathcal{I}_{4}^{2 \text {-loop }, \mathrm{P}}(s, u)\right. \\
+s^{2} \mathcal{I}_{4}^{2 \text {-loop }, \mathrm{NP}}(s, t)+s^{2} \mathcal{I}_{4}^{2 \text {-loop }, \mathrm{NP}}(s, u) \\
+ \text { cyclic }),
\end{gathered}
$$

where '+ cyclic' instructs one to add the two cyclic permutations of legs $(2,3,4)$, and $\mathcal{I}_{4}^{2 \text {-loop,P/NP }}$ are depicted in fig. 4.

We comment that using the two-loop amplitude (9), Green, Kwon and Vanhove 28 provided an explicit demonstration of the non-trivial M theory duality between $D=11$ supergravity and type II string theory. Here the finite parts of the amplitudes are most important, particularly their dependence on the radii of one or two compactified dimensions. 


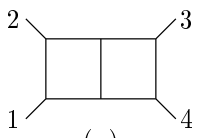

(a)

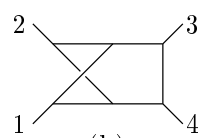

(b)
Figure 4. The planar (a) and non-planar (b) scalar integrals, $\mathcal{I}_{4}^{2 \text {-loop,P }}(s, t)$ and $\mathcal{I}_{4}^{2 \text {-loop,NP }}(s, t)$, appearing in the two-loop $N=8$ amplitudes. Each internal line represents a scalar propagator.

\subsection{Divergence Properties of $N=8$ Super- gravity}

Since the two-loop $N=8$ supergravity amplitude (9) has been expressed in terms of scalar integrals, it is straightforward to extract the divergence properties. The scalar integrals diverge only for $D \geq 7$; hence the two-loop $N=8$ amplitude is manifestly finite in $D=5$ and 6 , contrary to earlier expectations based on superspace power-counting arguments 17]. The discrepancy between the above explicit results and the earlier superspace power counting arguments may be understood in terms of an unaccounted higher dimensional gauge symmetry. Once this symmetry is accounted for, superspace power counting gives the same degree of divergence as the explicit calculation 29.

The manifest $D$-independence of the cutting algebra allowed us to extend the calculation to $D=11$, even though there is no corresponding $D=11$ super-Yang-Mills theory. The result (9) then explicitly demonstrates that $N=1 D=11$ supergravity diverges. In dimensional regularization there are no one-loop divergences so the first potential divergence is at two loops. The $D=11$ two-loop divergence may be extracted from the amplitude in eq. (9) yielding [1],

$$
\begin{aligned}
& \left.\mathcal{M}_{4}^{2 \text {-loop, } D=11-2 \epsilon}\right|_{\text {pole }}=\frac{1}{\epsilon(4 \pi)^{11}} \frac{1}{48} \frac{\pi}{5791500} \\
& \quad \times\left(438\left(s^{6}+t^{6}+u^{6}\right)-53 s^{2} t^{2} u^{2}\right) \\
& \quad \times\left(\frac{\kappa}{2}\right)^{6} \times s t u M_{4}^{\text {tree }} .
\end{aligned}
$$

Further work on the structure of the $D=11$ counterterm has been carried out in ref. [30].

Since the two-particle cut sewing equation iterates to all loop orders, one can compute all contributions which can be assembled solely from twoparticle cuts. (The five-loop integral in fig. 1, for example, falls into this category.) Counting powers of loop momenta in these contribu- tions suggests the simple finiteness formula, $L<$ $10 /(D-2)$ (with $L>1$ ), where $L$ is the number of loops. This formula indicates that $N=8$ supergravity is finite in some other cases where the previous superspace bounds suggest divergences [17], e.g. $D=4, L=3$. The first $D=4$ counterterm detected via the two-particle cuts of fourpoint amplitudes occurs at five, not three loops. (A recent improved superspace power count is, however, in agreement with this finiteness formula 29.) Further evidence that the finiteness formula is correct stems from the MHV contributions to $m$-particle cuts, in which the same supersymmetry cancellations occur as for the twoparticle cuts [1]. However, further work would be required to prove that other contributions do not alter the two-particle cut power counting. A related open question is whether one can prove that the five-loop divergence encountered in the two-particle cuts does not cancel against other contributions.

\section{Implications for the Einstein-Hilbert Lagrangian}

Consider the Einstein-Hilbert and Yang-Mills Lagrangians,

$L_{\mathrm{EH}}=\frac{2}{\kappa^{2}} \sqrt{-g} R, \quad L_{\mathrm{YM}}=-\frac{1}{4} F_{\mu \nu}^{a} F^{a \mu \nu}$.

The Einstein-Hilbert Lagrangian does not exhibit any obvious factorization property that might explain the KLT relations. We now outline some recent work [4] in interpreting the KLT relations in terms of the Lagrangians. (See also ref. [19].)

One of the key properties exhibited by the KLT relations (11) is the separation of graviton Lorentz indices into 'left' and 'right' sets. Consider the graviton field, $h_{\mu \nu}$. The KLT relations suggest that it should be possible to assign one Lorentz index as being associated with a 'left' gauge theory and the other to a 'right' one. Of course, since $h_{\mu \nu}$ is a symmetric tensor it does not matter which index is assigned to the left or to the right, but once the choice is made we would like to rearrange the gravity Lagrangian so that left indices only contract with left ones and right ones only with right ones. Using the KLT relations we can systematically find a Lagrangian with the desired properties order-by-order in the perturbative expansion by reversing the usual procedure of obtaining $S$ matrix elements from a Lagrangian. In ref. [- $\mid$, an appropriate field redefinition for connecting this Lagrangian to the standard Einstein-Hilbert one was described. 
In conventional gauges, the difficulty of factorizing the Einstein-Hilbert Lagrangian into left and right parts is already apparent in the kinetic terms. In de Donder gauge, the quadratic part of the Lagrangian is

$L_{2}=-\frac{1}{2} h_{\mu \nu} \partial^{2} h_{\mu \nu}+\frac{1}{4} h_{\mu \mu} \partial^{2} h_{\nu \nu}$,

so that the propagator is,

$P_{\mu \alpha ; \nu \beta}=$

$\frac{1}{2}\left[\eta_{\mu \nu} \eta_{\alpha \beta}+\eta_{\mu \beta} \eta_{\nu \alpha}-\frac{2}{D-2} \eta_{\mu \alpha} \eta_{\nu \beta}\right] \frac{i}{k^{2}+i \epsilon}$.

The appearance of the trace $h_{\mu \mu}$ in eq. (10) is unacceptable since it contracts a 'left' and 'right' index. (In Minkowski space, one of any two contracted indices should be raised using $\eta^{\mu \nu}$, but this is suppressed here.) Moreover, the propagator contains explicit dependence on the dimension $D$, which must somehow cancel from the tree-level $S$-matrix elements since there is no such dependence in the KLT relations or in the gauge theory amplitudes.

In order for the kinematic term to be consistent with the KLT equations, all terms which contract a 'left' Lorentz index with a 'right' one should be eliminated. In ref. [31] a dilaton field was introduced to allow for a field redefinition that removes the graviton trace from the quadratic terms in the Lagrangian. The appearance of the dilaton as an auxiliary field to help rearrange the Lagrangian is motivated by string theory which requires the presence of such a field. Following the discussion of refs. 31, [4, consider the Lagrangian for gravity coupled to a dilaton,

$L_{\mathrm{EH}}=\frac{2}{\kappa^{2}} \sqrt{-g} R+\sqrt{-g} \partial^{\mu} \phi \partial_{\mu} \phi$.

Since the auxiliary dilaton field is quadratic in the Lagrangian, it does not appear in any tree diagrams involving only external gravitons 伍.

It therefore does not alter the tree $S$-matrix of purely external gravitons. (For theories containing dilatons one can simply allow the dilaton to be an external physical state.) In de Donder gauge, for example, taking $g_{\mu \nu}=\eta_{\mu \nu}+\kappa h_{\mu \nu}$, the quadratic part of the Lagrangian including the dilaton is

$L_{2}=-\frac{1}{2} h_{\mu \nu} \partial^{2} h_{\mu \nu}+\frac{1}{4} h_{\mu \mu} \partial^{2} h_{\nu \nu}-\phi \partial^{2} \phi$.

The term involving $h_{\mu \mu}$ can be eliminated with the field redefinitions,

$h_{\mu \nu} \rightarrow h_{\mu \nu}+\eta_{\mu \nu} \sqrt{\frac{2}{D-2}} \phi$,
$\phi \rightarrow \frac{1}{2} h_{\mu \mu}+\sqrt{\frac{D-2}{2}} \phi$,

yielding

$L_{2} \rightarrow-\frac{1}{2} h_{\mu \nu} \partial^{2} h_{\mu \nu}+\phi \partial^{2} \phi$.

One might be concerned that the field redefinition (11) would alter the gravity $S$-matrix. However, the graviton $S$-matrix is guaranteed to be invariant under non-linear field redefinitions or under linear ones that do not alter the coupling to external traceless tensors.

Of course, the rearrangement of the quadratic terms is only the first step. In order to make the Einstein-Hilbert Lagrangian consistent with the KLT factorization a set of field variables should exist where all Lorentz indices can be separated into 'left' and 'right' classes. To do so, all terms of the form

$h_{\mu \mu}, \quad h_{\mu \nu} h_{\nu \lambda} h_{\lambda \mu}, \quad \cdots, \quad \operatorname{Tr}\left[h^{2 m+1}\right]$,

where $\operatorname{Tr}\left[h^{n}\right] \equiv h_{\mu_{1} \mu_{2}} h_{\mu_{2} \mu_{3}} \cdots h_{\mu_{n} \mu_{1}}$, need to be eliminated. A field redefinition which accomplishes this is,

$$
\begin{aligned}
g_{\mu \nu} & =e^{\sqrt{\frac{2}{D-2}} \kappa \phi} e^{\kappa h_{\mu \nu}} \\
& \equiv e^{\sqrt{\frac{2}{D-2}} \kappa \phi}\left(\eta_{\mu \nu}+\kappa h_{\mu \nu}+\frac{\kappa^{2}}{2} h_{\mu \rho} h_{\rho \nu}+\cdots\right) .
\end{aligned}
$$

As verified in ref. 何 through $\mathcal{O}\left(h^{6}\right)$, this choice eliminates all terms (12) which mix left and right Lorentz indices, even before gauge fixing.

It turns out that one can do better by performing further field redefinitions and choosing a particular non-linear gauge. With this gauge it is possible to express the off-shell three graviton vertex in terms of Yang-Mills three vertices [4],

$$
\begin{aligned}
& i G_{3}^{\mu \alpha, \nu \beta, \rho \gamma}(k, p, q) \\
& =-\frac{i}{2}\left(\frac{\kappa}{2}\right)\left[V_{\mathrm{GN}}^{\mu \nu \rho}(k, p, q) \times V_{\mathrm{GN}}^{\alpha \beta \gamma}(k, p, q)\right. \\
& \left.\quad+V_{\mathrm{GN}}^{\nu \mu \rho}(p, k, q) \times V_{\mathrm{GN}}^{\beta \alpha \gamma}(p, k, q)\right],
\end{aligned}
$$

where

$$
V_{\mathrm{GN}}^{\mu \nu \rho}(1,2,3)=i \sqrt{2}\left(k_{1}^{\rho} \eta^{\mu \nu}+k_{2}^{\mu} \eta^{\nu \rho}+k_{3}^{\nu} \eta^{\rho \mu}\right)
$$

is the color ordered Gervais-Neveu [32] YangMills three vertex, from which the color factor has been stripped. This is not the only possible 
reorganization of the three vertex which respects the KLT factorization. The main reason for using the above vertex is its simplicity.

This represents some initial steps in reorganizing the Einstein-Hilbert Lagrangian so that it respects the KLT relations. A complete derivation of the KLT expressions starting from the Einstein-Hilbert Lagrangian is, however, still lacking.

\section{Conclusions}

In this talk we described progress in understanding perturbative quantum gravity thorough use of the Kawai-Lewellen-Tye string relations [6] and D-dimensional unitarity [7,8]. This provides an alternative way to perturbatively quantize gravity theories, without direct reference to their Lagrangians or Hamiltonians, and leads to a relatively efficient organization of the gravity $S$ matrix. Known gauge theory tree amplitudes can be recycled into gravity tree amplitudes which can then be recycled into gravity loop amplitudes.

Using this approach, we described the argument of ref. [1] that $D=4 N=8$ supergravity is less divergent than had been previously believed. Moreover, we outlined the computation of the two-loop four-point amplitude in maximal supergravity given in ref. [1]. Using this result, it is straightforward to show that $N=1 D=11$ supergravity diverges and is not protected by any 'magic' of M theory. (Further elaboration of the structure of the counterterm may be found in ref. 30.) The two-loop supergravity amplitude (9) has also been used by Green, Kwon and Vanhove [28] to verify a non-trivial M theory duality.

There are a number of interesting open questions. For example, the methods described here have been used to investigate only maximal supergravity. It would be interesting to systematically re-examine the divergence structure of nonmaximal theories. (Some very recent work on this may be found in ref. 33.) Using the methods described in this talk it might, for example, be possible to systematically determine finiteness conditions order-by-order in the loop expansion. A direct derivation of the Kawai-Lewellen-Tye decomposition of gravity amplitudes in terms of gauge theory ones starting from the Einstein-Hilbert Lagrangian perhaps would lead to a useful reformulation of gravity. Connected with this is the question of whether the heuristic notion that gravity is the square of gauge theory can be given meaning outside of perturbation theory. It would also be interesting to know whether it is possible to relate more general solutions of the classical equations of motion for gravity to those for gauge theory.

In summary, in this talk we discussed how the

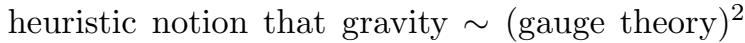
can be exploited to develop a better understanding of perturbative gravity.

\section{REFERENCES}

1. Z. Bern, L. Dixon, D.C. Dunbar, M. Perelstein and J.S. Rozowsky, Nucl. Phys. B530 (1998) 401, hep-th/9802162.

2. Z. Bern, L. Dixon, M. Perelstein and J. S. Rozowsky, Phys. Lett. B444 (1998) 273, hepth/9809160.

3. Z. Bern, L. Dixon, M. Perelstein and J. S. Rozowsky, Nucl. Phys. B546 (1999) 423, hepth/9811140

4. Z. Bern and A.K. Grant, Phys. Lett. B457 (1999) 23, hep-th/9904026.

5. Z. Bern, A. De Freitas and H. L. Wong, preprint hep-th/9912033.

6. H. Kawai, D.C. Lewellen and S.-H.H. Tye, Nucl. Phys. B269 (1986) 1.

7. Z. Bern, L. Dixon, D.C. Dunbar and D.A. Kosower, Nucl. Phys. B425 (1994) 217, hepph/9403226]; Z. Bern, L. Dixon, D.C. Dunbar and D.A. Kosower, Nucl. Phys. B435 (1995) 59, hep-ph/9409265; Z. Bern and A. G. Morgan, Nucl. Phys. B467 (1996) 479, hep-ph/9511336; Z. Bern, L. Dixon, D.A. Kosower and S. Weinzierl, Nucl. Phys. B489 (1997) 3, hep-ph/9610370]; Z. Bern, L. Dixon and D.A. Kosower, Nucl. Phys. B513 (1998) 3, hep-ph/9708239; J.S. Rozowsky, preprint hep-ph/9709423; Z. Bern, L. Dixon and D. A. Kosower, JHEP 0001 (2000) 27, [hep-ph/0001001].

8. Z. Bern, L. Dixon and D.A. Kosower, Ann. Rev. Nucl. Part. Sci. 46 (1996) 109, hep$\mathrm{ph} / 9602280$.

9. G. 't Hooft and M. Veltman, Annales Poincaré Phys. Theor. A20 (1974) 69.

10. G. 't Hooft, Nucl. Phys. B62 (1973) 444.

11. S. Deser and P. van Nieuwenhuizen, Phys. Rev. D10 (1974) 401; Phys. Rev. D10 (1974) 411; S. Deser, H. Tsao and P. van Nieuwenhuizen, Phys. Rev. D10 (1974) 3337.

12. M.H. Goroff and A. Sagnotti, Nucl. Phys. B266 (1986) 709; A.E.M. van de Ven, Nucl. Phys. B378 (1992) 309.

13. M.T. Grisaru, H.N. Pendleton and P. van Nieuwenhuizen, Phys. Rev. D15 (1977) 996; M.T. Grisaru and H.N. Pendleton, Nucl. Phys. B124 (1977) 81; S.J. Parke and 
T.R. Taylor, Phys. Lett. B157 (1985) 81.

14. M.T. Grisaru, P. van Nieuwenhuizen and J.A. Vermaseren, Phys. Rev. Lett. 37 (1976) 1662.

15. M.T. Grisaru, Phys. Lett. 66B (1977) 75; E. Tomboulis, Phys. Lett. 67B (1977) 417.

16. R.E. Kallosh, Phys. Lett. B99 (1981) 122.

17. S. Deser, J.H. Kay and K.S. Stelle, Phys. Rev. Lett. 38 (1977) 527; P.S. Howe, K.S. Stelle amd P.K. Townsend, Nucl. Phys. B191 (1981) 445; P.S. Howe and K.S. Stelle, Phys. Lett. B137 (1984) 175; Int. J. Mod. Phys. A4 (1989) 1871.

18. B.S. DeWitt, Phys. Rev. 162 (1967) 1239; M. Veltman, in Les Houches 1975, Proceedings, Methods In Field Theory (Amsterdam 1976).

19. W. Siegel, Phys. Rev. D47 (1993) 5453, hepth/9302036|; Phys. Rev. D48 (1993) 2826, hep-th/9305073; in Proceedings of Strings 1993, eds. M.B. Halpern, A. Sevrin and G. Rivlis (World Scientific, Singapore, 1994), hep-th/9308133.

20. F.A. Berends, W.T. Giele and H. Kuijf, Phys. Lett. B211 (1988) 91.

21. S.J. Parke and T.R. Taylor, Phys. Rev. Lett. 56 (1986) 2459.

22. M. Mangano and S.J. Parke, Phys. Rep. 200 (1991) 301; L. Dixon, in Proceedings of Theoretical Advanced Study Institute in Elementary Particle Physics (TASI 95), ed. D.E. Soper, hep-ph/9601359.

23. W.L. van Neerven, Nucl. Phys. B268 (1986) 453.

24. D.C. Dunbar and P.S. Norridge, Nucl. Phys. B433 (1995) 181, hep-th/9408014; D.C. Dunbar and P.S. Norridge, Class. Quant. Grav. 14 (1997) 351, hep-th/9512084.

25. Z. Bern, G. Chalmers, L. Dixon and D. A. Kosower, Phys. Rev. Lett. 72 (1994) 2134, hep-ph/9312333. Z. Bern and G. Chalmers, Nucl. Phys. B447 (1995) 465, hep-ph/9503236.

26. Z. Bern, J.S. Rozowsky and B. Yan, Phys. Lett. B401 (1997) 273; hep-ph/9702424]; in proceedings of the 5th International Workshop on Deep Inelastic Scattering and QCD (DIS 97), Chicago, IL, 14-18 Apr 1997, hepph/9706392

27. M.B. Green, J.H. Schwarz and L. Brink, Nucl. Phys. B198 (1982) 474.

28. M.B. Green, H. Kwon and P. Vanhove, hepth/9910055.

29. M. Petrin, P.S. Howe and K.S. Stelle, to appear.
30. S. Deser and D. Seminara, Phys. Rev. Lett. 82 (1999) 2435, hep-th/9812136]; D. Seminara, preprint hep-th/0001028.

31. Z. Bern, D.C. Dunbar and T. Shimada, Phys. Lett. B312 (1993) 277, hep-th/9307001.

32. J.L. Gervais and A. Neveu, Nucl. Phys. B46 (1972) 381.

33. D. C. Dunbar, B. Julia, D. Seminara and M. Trigiante, preprint hep-th/9911158. 